\title{
Luminescent Polymer Optical Fibre Sensor for Temperature Measurement
}

\author{
P. Miluski*, D. Dorosz, J. Żmojda, M. Kochanowicz and J. Dorosz \\ Białystok University of Technology, Wiejska 45D, 15-351 Białystok, Poland \\ (Received May 12, 2014; revised version January 20, 2015; in final form February 4, 2015)
}

\begin{abstract}
In the paper the luminescence optical fiber used as a temperature sensor is presented. The new construction of rhodamine B doped, polymethyl methacrylate optical fibre with silver coating is shown. The fabrication process and luminescent properties of rhodamine B in sensing fibre are investigated. The attenuation and spectral shift of luminescence peak vs. the fibre length were measured and used for optimization of sensor construction. The characteristics of sensor for temperature range from 293 up to $343 \mathrm{~K}$ are shown. The relative sensitivity equal to $-5.4 \times 10^{-4} \mathrm{~K}^{-1}$ was obtained. The article presents also the potential applications of presented sensor.
\end{abstract}

DOI: 10.12693 /APhysPolA.127.730

PACS: 42.81.-i, 42.81.Wg, 42.79.-e, 42.70.Jk

\section{Introduction}

The well known electrical resistance and thermocouple based sensors are used in many fields of industrial and science applications. They assure high accuracy and repeatability of measurements, but also have some disadvantages such as electrical connection to sensing element and non immunity to strong electromagnetic fields. The use of optical radiation for temperature detection can eliminate the above drawbacks. The advantages of optical fibers e.g. small size, galvanic isolation cause that they are used in many special applications for industry and environmental monitoring. The pyrometric methods, based on emission spectrum analysis, allows to non contact temperature measurement typically up to $3500 \mathrm{~K}$ [1-3]. The two channel embedded infrared optical fibre measurement system, based on emission spectrum of silver halides membrane, was presented in literature [4]. Other sensor constructions based on absorption spectrum changes of GaAs crystal vs. the temperature were also investigated $[5,6]$. The well known and predictable changes of semiconductor band gap energy can be detected using absorption spectrum (typically its edge shifts about $0.4 \mathrm{~nm} / \mathrm{K}$ ). The temperature measurement is also possible using thermochromic effect which involves change in the position of individual atoms in the crystalline lattice. The phenomenon is observed in many substances e.g. salts $\mathrm{Cu}_{2} \mathrm{HgI}_{4}$ and aqueous-alcoholic solution of cobalt chloride $[7,8]$. The novel polymeric materials can exhibit thermochromic properties themselves e.g. polyacetylenes, polydiacetylenes, polythiophenes and polyanilines. The conjugated polymers can be doped by organic leuco dyes and inorganic pigments in microencapsulation processes. These hybrid organicinorganic materials allow to obtain high sensitivity of measurements [9-11]. The temperature effect on birefringence dispersion in capillary and microstructure fibers

* corresponding author; e-mail: p.miluski@pb.edu.pl filled by nematic liquid crystals was also investigated in literature [12-15]. One of the most accurate method is fiber Bragg gratings (FBG) structure in glass optical fibre [16-18].

The grating optical fibre sensors assure very high sensitivity but they are simultaneously non immune to mechanical expansion phenomenon and very fragile. The high sensitivity and repeatable temperature measurements can be obtained using luminescence phenomenon. The new luminescent materials (doped solgel thin films, porous ceramics and rare earth doped luminescent glasses) cause growing interest of luminescent based sensing applications. The numerous applications of temperature luminescent sensors based on glass host are presented in literature [19-23]. These application assure high optical damage threshold and long term stability but the sensitivity is typically much lower than organic doped polymeric materials. The fabrication process of sensing structure is also more difficult than polymers. The organic luminescent optical sensors have a lot of advantages e.g. high quantum yield and luminescence, high sensitivity, low cost of materials and treatment. This causes the wide range of application of polymeric materials for sensors [24-31]. The well known group of luminescent organic dyes are xanthenes. They are widely used because their high quantum yield, low photobleaching effect (especially rhodamine B) and good solubility in organic solvents.

Presented in the paper construction of temperature sensor is based on rhodamine $\mathrm{B}$ doped polymethyl methacrylate (PMMA) fibre. The organic dyes in polymer host allows to obtain the efficient luminescence in visible spectrum range [28-30]. The PMMA also does not interact with the rhodamine molecules and does not significantly affect on quantum yield, absorption, emission and decay time. Their potential applications have although some restrictions caused by photobleaching effect and low glass transition temperature. The proposed doped polymer fibre temperature sensor assures high output signal and sensitivity in the temperature range from 293 up to $343 \mathrm{~K}$. 


\section{Construction of sensor}

The energy levels of organic molecules can be described as broad bands caused by vibrational and thermal relaxation of dye's molecule. The rhodamine B has high crosssection and this assures high luminescence and possibility of signal amplification for short distance in laser applications. The most of organic luminescent dyes doped polymer fibers have typical core/cladding structure with doped core region. According to optical fibre aperture only a part of luminescence signal is guided in optical fibre structure. This kind of construction is also sensitive to ambient sources of light. This effect is undesired especially for sensing applications. The proposed construction is based on silver coated PMMA fibre doped by rhodamine $\mathrm{B}$. The waveguide is fabricated using mirror structure which assures immunity to ambient sources of light and prevents pumping and luminescence signal leakage from fibre.

It is important to fulfill safety operating condition with lasers. It is known that mirror guiding structure has higher signal attenuation compared to typical optical fibres. This effect limits the maximum sensing length of the fibre. The structure and photo of luminescent polymer fibre used for sensor fabrication is presented in Fig. 1. The PMMA doped preform was fabricated using thermal polymerization method. The preform (polymer rod with diameter $11 \mathrm{~mm}$ ) was fabricated in glass test tubes. The rhodamine $\mathrm{B}$ concentration used for fibre fabrication was $20 \times 10^{-4} \mathrm{~mol} / \mathrm{l}$. The chosen dye concentration assures efficient optical pump absorption and exhibit high luminescence. The concentration of organic dye was low enough that the fabricated preform was transparent. The polymer fibre (diameter $0.5 \mathrm{~mm}$ ) was fabricated from polymer rod using drawing process. The fibre surface was coated using mirror silvering method based on reduction of silver nitrate solution. The additional polyurethane coating was used for mechanical protection of the fibre.

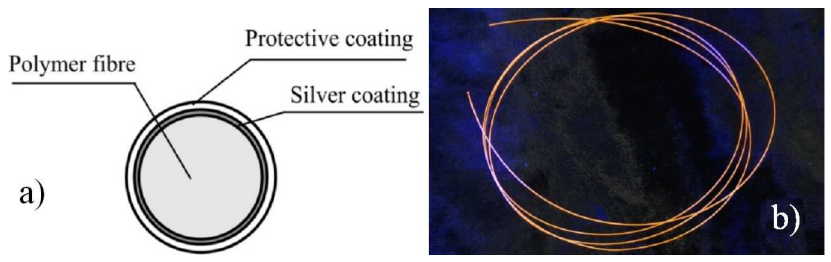

Fig. 1. (a) The cross section of silver coated sensing fibre, (b) the photo of rhodamine B doped fibre used for sensor fabrication.

The diode pumped solid state laser with emission $532 \mathrm{~nm}(\mathrm{FWHM}=10 \mathrm{~nm})$ and $200 \mathrm{~mW}$ optical power was used for excitation of face of the fibre. The excitation laser spectrum is in good agreement with absorption spectrum of rhodamine B molecule and assures short efficient optical pumping length. The emission spectrum at end face of fibre were measured using Stellarnet Green Wave spectrometer. The spectra were recorded in the range of 550-800 nm with $1 \mathrm{~nm}$ resolution. The used concentration of rhodamine $\mathrm{B}$ allows to high luminescence emission with maximum in the range from 622 up to $665 \mathrm{~nm}$. Although the attenuation and reabsorption effects cannot be omitted, the luminescence characteristic vs. the length of fibre were measured. It was experimentally check that the absorption length of laser signal is about $60 \mathrm{~mm}$. The luminescence spectra were recorded for fibre length from 30 up to $150 \mathrm{~mm}$. The attenuation and red shift of maximum peak was observed. The measurements results are presented in Figs. 2, 3.

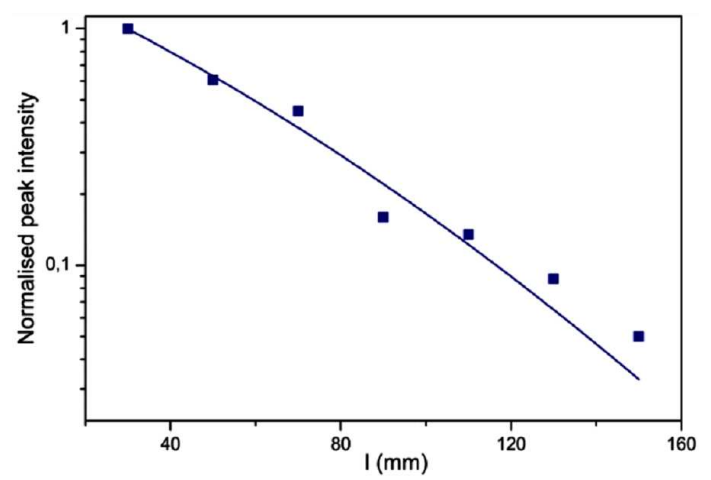

Fig. 2. The luminescence peak intensity of silver coated fibre vs. its length.

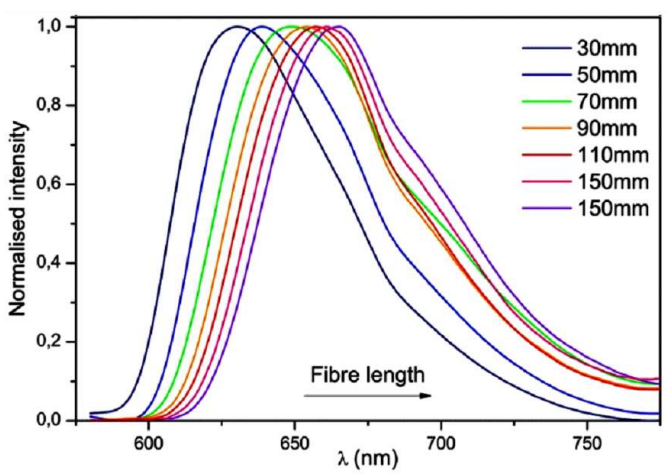

Fig. 3. The normalised luminescence spectrum of silver coated doped PMMA fibre (diameter $0.5 \mathrm{~mm}$, excitation wavelength $532 \mathrm{~nm}$, fibre length $30,50,70,90$, $110,130,150 \mathrm{~mm})$.

The measured attenuation of fabricated fibre is $80 \mathrm{~dB} / \mathrm{m}$. The spectral red shift effect is presented in Figs. 3, 4. The luminescence spectrum shows significant spectral red shift vs. the well known maximum luminescence peak for rhodamine B in water solution (about $580 \mathrm{~nm}$ ). The parameters investigated in presented structure were: nonlinear effects for high optical power radiation, signal reabsorption and high attenuation increasing with dye concentration. The influence of concentration of dye for spectral shape movement was presented in literature [31]. The luminescence exhibits red shift for high dye concentration. The theoretical model of attenuation and spectral shift mechanism for rhodamine B doped step index PMMA optical fibre were described in $[32,33]$. Authors proposed theoretical model 
of $\lambda$-dependent absorption and emission cross-sections in optical fibre structure. It was shown that reabsorption effect and high attenuation of luminescence peak for high concentration of rhodamine $B$ are the main factors of spectral shift. The luminescence spectrum width (FWHM) can be tuned, in fabricated fibre, in the range from 65 up to $77 \mathrm{~nm}$ by changing the optical fibre length. The presented results are in good agreement with data reported in literature [31-33]. The $150 \mathrm{~mm}$ fibre length was chosen for sensor construction as it assures high optical coupling level and luminescence signal.

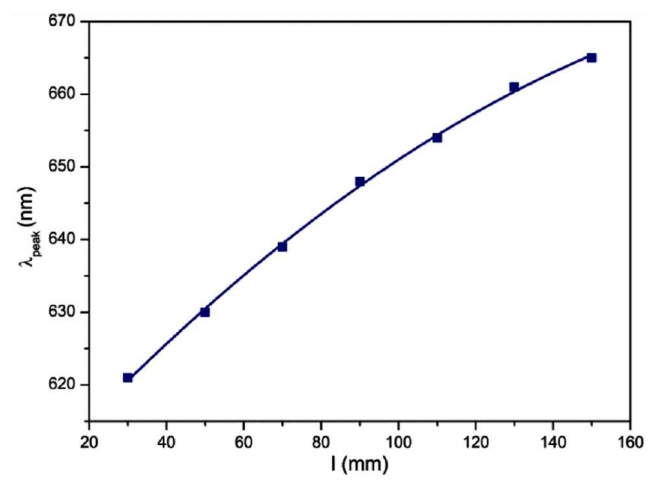

Fig. 4. The maximum peak wavelength of silver coated doped PMMA fibre vs. its length (fibre diameter $0.5 \mathrm{~mm}$, excitation wavelength $532 \mathrm{~nm}$ ).

\section{Measurements}

The scheme of measurement system is presented in Fig. 5. The solid state laser with emission $532 \mathrm{~nm}$ was used as excitation source. The $10 \mathrm{~s}$ excitation pulses were used during experiments to prevent the photobleaching effects. The emission spectra at end tip of fibre were measured using spectrometer. The fibre was attached to aluminum plate and thermally heated using $40 \mathrm{~W}$ Peltier module and temperature controller FOX-1004 with hysteresis $\pm 1 \mathrm{~K}$.

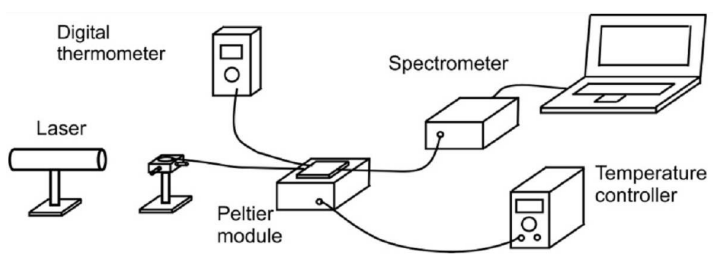

Fig. 5. The schematic of measurement system.

The system was prevented from ambient sources of light. The recorded spectra are presented in Fig. 6 . The temperature range was chosen from 293 up to $343 \mathrm{~K}$. The lower temperature limitation is caused by nonlinear characteristic of maximum peak of luminescence. The high temperature range limitation is caused by PMMA glass transition temperature and output signal intensity. Measured temperature dependency characteristic is presented in Fig. 7.

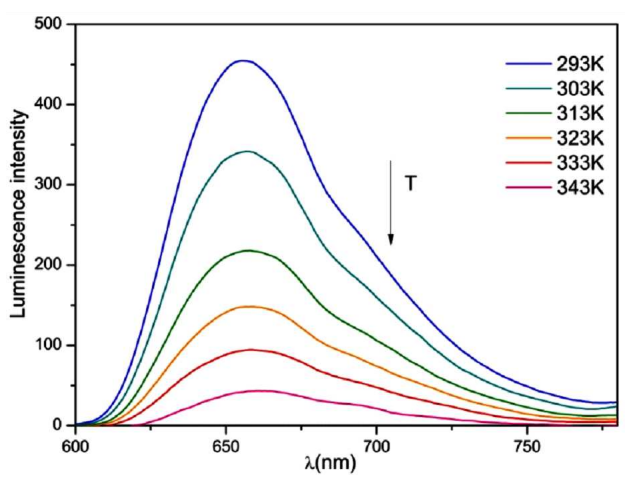

Fig. 6. The recorded spectra of fabricated sensor vs. temperature.

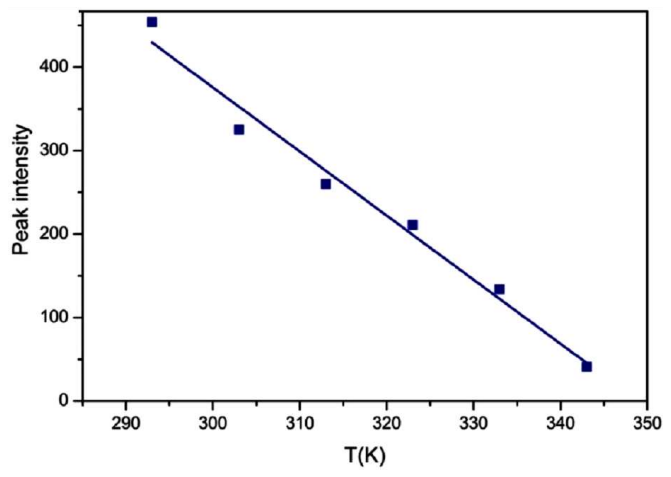

Fig. 7. The experimentally obtained characteristic of fabricated sensor vs. temperature.

The useful part of characteristic is contained between 293 and $343 \mathrm{~K}$. The temperature sensitivity of sensor can be calculated using equation:

$$
S=\frac{\mathrm{dR}}{\mathrm{d} T},
$$

where the $R$ is peak intensity and $T$ is measured temperature. The experiments have shown that the polymer fibre exhibits high slope of maximum luminescence peak vs. temperature. However the value of relative sensitivity of sensor $S_{\mathrm{R}}$ better describes the properties of sensor. $S_{\mathrm{R}}$ was calculated using equation

$$
S_{\mathrm{R}}=\frac{1}{R} \frac{\mathrm{d} R}{\mathrm{~d} T} .
$$

The obtained characteristic of relative sensitivity $S_{\mathrm{R}}$ is shown in Fig. 8.

The slope of relative sensitivity for linear fitting is equal to $-5.4 \times 10^{-4} \mathrm{~K}^{-1}(R a=0.89)$. The photobleaching effects for long term measurement application cannot be omitted and should be investigated. The commercial application of presented sensor should be predicted by temperature and laser excitation tests. The advantages of fabricated material such as high sensitivity, low weight and high environmental compatibility cause that this kind of construction can be used in special sources of light and sensor technology. The silver mirror layer assures immunity to ambient sources of light. The small size caused that sensor can be incorporated 


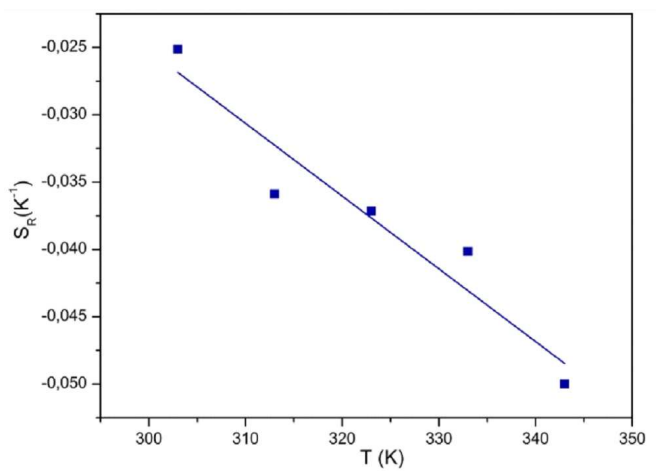

Fig. 8. The relative sensitivity of fabricated optical temperature sensor.

into the structure of composite materials during fabrication process. This kind of sensors can be useful in many applications in moderate temperature range monitoring in industry e.g. composite reinforcement materials, environmental temperature monitoring or small size distributed sensors.

\section{Summary}

The fabrication process of silver coated rhodamine $\mathrm{B}$ doped PMMA fibre was presented. The maximum intensity and spectral luminescence shift in polymer fibre was shown. The temperature (from 293 up to $343 \mathrm{~K}$ ) characteristic of designed temperature sensor was experimentally obtained. The relative sensitivity of sensor is equal to $-5.4 \times 10^{-4} \mathrm{~K}^{-1}$. The maximum temperature limitation caused by PMMA glass transition temperature $T_{\mathrm{g}}=378 \mathrm{~K}$ can be overcome by using other polymer matrices e.g. cyclo-olefin polymers (COP) with $T_{\mathrm{g}}$ up to $413 \mathrm{~K}$ or polycarbonate $(\mathrm{PC}) T_{\mathrm{g}}$ about $423 \mathrm{~K}$. Future work will include new polymer matrices for this purposes. The presented sensor can be considered for the applications in automation under the investigated temperature range.

\section{Acknowledgments}

This work was supported by Białystok University of Technology, project no. S/WE/4/2013.

\section{References}

[1] N.A. Riza, M. Sheikh, IEEE Sens. J. 10, 219 (2010).

[2] I.Y. Orlov, I.A. Nikiforov, A.V. Afanasjev, Wireless Eng. Technol. 5, 25 (2014).

[3] J.E. Polk, C.M. Marrese-Reading, B. Thornber, L. Dang, L.K. Johnson, I. Katz, Rev. Sci. Instrum. 78, 093101 (2007).

[4] J.Y. Wook, W.J. Kyoung, K.S. Jeong, M. Jinsoo, H. Ki-Tek, P. Jang-Yeon, G.P. Byung, L. Bongsoo, Sensors 11, 9549 (2011).

[5] K. Uesugi, I. Suemune, T. Hasegawa, T. Akutagawa, T. Nakamura, Appl. Phys. Lett. 76, 1285 (2000).

[6] R. Pässler, Phys. Status Solidi 236, 710 (2003).

[7] S. Kałuża, Z. Szcześniak, Metrol. Measur. Syst. 12, 285 (2005).
[8] N. Carmona, E. Herrero-Hernandez, J. Llopis, M.A. Villegas, Col-Gel Sci. Technol. 47, 31 (2008).

[9] L. Ji-Seok, L. Joo Seop, P. Hyun Wook, L. Cheol Hee, A. Dong June, K. Jong-Man, NSTI-Nanotech. 1, 485 (2005).

[10] A. Chowdhury, M. Joshi, B.S. Butola, J. Eng. Fibers Fabrics 9, 107 (2014).

[11] A. Seeboth, D. Lötzsch, Thermochromic Phenomena in Polymers, Smithers, Rapra 2008.

[12] Z. Mykytyuk, V. Ivanytskyy, V. Cherpak, D. Bolormaa, A. Fechan, Opto-Electron. Rev. 10, 79 (2002).

[13] S. Shaik, A. Desai, Int. J. Interdisciplin. Multidisciplin. Studies 1, 103 (2014).

[14] Y.A. Nastishin, H. Liu, T. Schneider, V. Nazarenko, R. Vasyuta, S.V. Shiyanovskii, O.D. Lavrentovich, Phys. Rev. E 72, 041711 (2005).

[15] A.A. Zaki, J. Mod. Phys. 4, 517 (2013).

[16] P. Saidi Reddy, R.L.N. Sai Prasad, K. Srimannarayana, M. Sai Shankar, D. Sen Gupta, Opt. Appl. 40, 685 (2010).

[17] I. Toccafondo, M. Taki, A. Signorini, F. Zaidi, T. Nannipieri, S. Faralli, F. Pasquale, Opt. Lett. 37, 4434 (2012).

[18] T.A. Hamdalla, M.Y. El-Bakry, M.A. Moussa, Int. J. Sci. Eng. Res. 3, 351 (2012).

[19] A.K. Singh, Sens. Actuat. A 136, 173 (2007).

[20] J. Żmojda, D. Dorosz, M. Kochanowicz, J. Dorosz, Proc. SPIE 8010, 80100M (2011).

[21] M. Kochanowicz, D. Dorosz, J. Zmojda, P. Miluski, J. Dorosz, Acta Phys. Pol. A 124, 471 (2013).

[22] M.G. Cidália, J.H. Andrew, J.Q. Susan, G. Thorfinnur, Coord. Chem. Rev. 252, 2512 (2008).

[23] P. Jorge, M.A. Martins, T. Trindade, J.L. Santos, F. Farahi, Sensors 7, 3489 (2007).

[24] L. Yu-Lung, C. Chen-Shane, Y. Jiahn-Piring, C. Yuan-Che, Sens. Actuat. B 131, 479 (2008).

[25] L. Bilro, N. Alberto, J.L. Pinto, R. Nogueira, Sensors 12, 12184 (2012).

[26] C. Baleizao, S. Nagl, M. Schaferling, M.N. BerberanSantos, O.S. Wolfbeis, Anal. Chem. 80, 6449 (2008).

[27] N. Tomczak, D. Jańczewski, Mingyong Hana, G. Julius Vancsoa, Progr. Polym. Sci. 34, 393 (2009).

[28] N.R. Tanzeela, M.R. Brouwer, Adv. Fluoresc. Rep. Chem. Biol. III Springer Ser. Fluoresc. 113, 91 (2011).

[29] P. Miluski, D. Dorosz, M. Kochanowicz, J. Żmojda, Proc. SPIE 8903, 89030C-6 (2013).

[30] C. Pietsch, A. Vollrath, R. Hoogenboom, U.S. Schubert, Sensors 10, 7979 (2010).

[31] C.V. Bindhu, S.S. Harilal, G.K. Varier, R.C. Issac, V.P.N. Nampoori, C.P.G. Vallabhan, J. Phys. D Appl. Phys. 29, 1074 (1996).

[32] J. Arrue, F. Jiménez, I. Ayesta, M. Asunción Illarramendi, J. Zubia, Polymers 3, 1162 (2011).

[33] H.Y. Tama, Chi-Fung Jeff Pun, G. Zhou, X. Cheng, M.L.V. Tse, Opt. Fiber Technol. 16, 357 (2010). 